Reprod. Nutr. Dévelop., 1987, 27 (1 B), 301-302.

\title{
Influence du niveau énergétique et azoté de la ration sur l'aminoacidémie libre de vaches laitières en début de lactation
}

\author{
C. CHAMPREDON, B. REMOND $\left({ }^{*}\right)$, P. PATUREAU-MIRAND
}

Laboratoire d'Etude du Métabolisme Azoté,

(*) Laboratoire de la Lactation,

I.N.R.A., Theix, 63122 Ceyrat, France.

Summary. The effects of energy (UFL) and nitrogen (PDI) intakes on blood free amino acids, glucose and urea were studied in cows in early lactation. Alanine, urea and isoleucine were highly related $(P<0.01)$ to energy level and urea, valine, isoleucine, leucine and methionine to nitrogen level. Methionine was negatively correlated to nitrogen intake, assuming that this amino acid might be the first limiting one in the PDI supply.

Les niveaux d'apports alimentaires, énergétique et surtout azoté, influencent la production laitière chez les vaches en début de lactation. Nous avons voulu savoir dans quelle mesure les variations de l'aminoacidémie libre après le vêlage reflètent les modifications métaboliques liées à celles de l'état nutritionnel.

Matériel et méthodes. Dans une expérience de type factoriel $2 \times 2$, 45 vaches laitières alimentées individuellement et réparties en 4 lots de 10 à 12 animaux, ont reçu une ration à base d'ensilage de maïs complémenté par des quantités variables d'aliments concentrés ayant, selon les lots, des teneurs en PDI différentes en fonction de la proportion de tourteaux (soja + colza) traités au formol qu'ils renferment (Journet et al., 1983 - Essai 1). Deux niveaux d'apports énergétiques, l'un élevé : $10,1,14,0$ et 16,9 UFL/j respectivement en 1 re (stade 1 ), $3^{e}$ (stade 2 ) et $6^{e}$ (stade 3 ) semaine de lactation, l'autre faible : 9,4, 12,3 et 14,7, étaient associés à 2 niveaux d'apports azotés : 1467,1839 et $1871 \mathrm{~g}$ de $\mathrm{PDI} / \mathrm{j}$ pour l'un ; 1030,1402 et $1648 \mathrm{~g}$ pour l'autre. Des prélèvements de sang jugulaire étaient effectués $3 \mathrm{~h}$ après la distribution du repas du matin à la fin des $1^{r e}, 3^{e}$ et $6^{e}$ semaines de lactation afin de déterminer l'aminoacidémie libre, l'urémie et la glycémie (Champredon et al., 1977) sur un échantillon moyen du sang des animaux du même lot. La concentration de ces paramòtres sanguins a été étudiée par une analyse de variance-covariance dans laquelle nous avons introduit simultanément l'apport énergétique (UFL) et l'apport azoté (PDI) comme covariables et la semaine de lactation comme facteur.

Résultats. Les apports élevés en azote ont permis de satisfaire les besoins estimés en PDI, dès la première semaine de lactation, alors que les apports énergétiques les plus importants n'ont permis de couvrir les besoins en UFL qu'au cours de la $6^{e}$ semaine. C'est avec les rations les plus riches en azote que l'on observe les productions laitières les plus fortes (tabl. 1).

Le niveau élevé en énergie (tabl. 1) accroît l'alanimémie et abaisse l'urémie et les teneurs en isoleucine et en valine. Les effets de l'apport énergétique expliquent l'évolution de ces paramètres entre la $1^{\text {re }}$ et la $6^{\circ}$ semaine de lactation : élévation de la concentration en alanine (de 1,18 a $1,55 \mathrm{mg} / 100 \mathrm{~g}$ ) et diminution de celles en urée $(31$ à 24$)$, valine $(2,18$ à 1,44$)$ et isoleucine $(1,31$ à 0,93$)$. Ces variations sont une des manifestations, sur le plan métabolique, de l'amélioration du degré de satisfaction des besoins en substrats glucoformateurs. L'augmentation de l'alaninémie et la diminution de l'urémie pourraient correspondre à une réduc- 
tion de la gluconéogenèse à partir des acides aminés lorsque les apports énergétiques sont les plus élevés.

TABL. 1. - Effets déterminés par l'analyse de variance-covariance de l'apport énergétique, de l'apport azoté et du stade de lactation sur la production de lait à $4 \%$ de matières grasses ( $\mathrm{kg} / \mathrm{jour}$ ), les teneurs en urée, glucose et acides aminés libres du sang $(\mathrm{mg} / 100 \mathrm{~g})$.

\begin{tabular}{|c|c|c|c|c|c|c|c|c|}
\hline & \multirow{2}{*}{$\begin{array}{l}\text { Valeurs } \\
\text { moyennes }\end{array}$} & \multirow{2}{*}{$\begin{array}{c}\text { Effet } \\
\text { energie }\left({ }^{1}\right)\end{array}$} & \multirow{2}{*}{$\begin{array}{c}\text { Effet } \\
\text { azote }\left({ }^{2}\right)\end{array}$} & \multicolumn{4}{|c|}{ Effet stade } & \multirow{2}{*}{ ETR $\left.\left.\right|^{3}\right\rangle$} \\
\hline & & & & S1 & S2 & S3 & Sign. & \\
\hline Lait $4 \%$ & 24,37 & $-0,28 \mathrm{~ns}$ & $+0,76^{* *}$ & $-2,51$ & $+1,71$ & $+0,80$ & ${ }^{*}$ & 1,30 \\
\hline Thréonine & 1,39 & $+0,08 \mathrm{~ns}$ & $+0,02 \mathrm{~ns}$ & $-0,34$ & $-0,10$ & +0.44 & ns & 0,18 \\
\hline Alanine & 1,42 & $+0,21^{* *}$ & $-0,04^{*}$ & $+0,29$ & $+0,10$ & $-0,39$ & * & 0,10 \\
\hline Valine & 1,91 & $-0,24^{*}$ & $+0,18^{* *}$ & $+0,06$ & $+0,12$ & $-0,18$ & ns & 0,19 \\
\hline Méthionine & 0,32 & $+0,03^{*}$ & $-0,02^{* *}$ & $+0,06$ & $-0,01$ & $-0,05$ & $\mathrm{~ns}$ & 0,03 \\
\hline Isoleucine & 1,13 & $-0,21^{* *}$ & $+0,12^{* *}$ & $-0,11$ & $-0,02$ & $+0,12$ & ns & 0,14 \\
\hline Leucine & 1,67 & $-0,05 \mathrm{~ns}$ & $+0,11^{* *}$ & $+0,37$ & $-0,02$ & $-0,35$ & ns & 0,13 \\
\hline Phénylalanine & 0,72 & $0,00 \mathrm{~ns}$ & $+0,02 \mathrm{~ns}$ & $+0,10$ & $-0,01$ & $-0,09$ & ns & 0,06 \\
\hline Lysine & 1,06 & $-0,03 \mathrm{~ns}$ & $+0,03^{*}$ & $-0,03$ & $+0,18$ & $-0,15$ & $* *$ & 0,06 \\
\hline Histidine & 1,33 & $-0,02 \mathrm{~ns}$ & $+0,05^{*}$ & $+0,03$ & $+0,26$ & $-0,29$ & ** & 0,10 \\
\hline Arginine & 0,42 & $0,00 \mathrm{~ns}$ & $+0,01 \mathrm{~ns}$ & $-0,03$ & $+0,12$ & $-0,09$ & ** & 0,04 \\
\hline 3-M. Histidine & 0,10 & $+0,01 \mathrm{~ns}$ & $+0,01 \mathrm{~ns}$ & $+0,07$ & $-0,04$ & $-0,03$ & ns & 0,03 \\
\hline Urée & 27,17 & $-7,03^{* *}$ & $+3,46^{* *}$ & $-8,77$ & $-1,15$ & $+9,92$ & ** & 1,89 \\
\hline Glucose & 61,75 & $+2,12 \mathrm{~ns}$ & $-0,60 \mathrm{~ns}$ & $-1,10$ & $+0,70$ & $+0,40$ & ns & 3,56 \\
\hline
\end{tabular}

(1) Effet énergie : résultat d'une augmentation de l'ingestion de 1 UFL. ( $^{2}$ ) Effet azote : résultat d'une augmentation de l'ingestion de $100 \mathrm{~g}$ de PDI. $\left(^{3}\right)$ Ecart-type résiduel. - Signification des effets énergie azote et stade : ns : non significatif; ${ }^{*}: \mathrm{P}<0,05 ;{ }^{* *}: \mathrm{P}<0,01$.

L'élévation des quantités de PDI ingérées se traduit par un accroissement des teneurs sanguines en urée et en acides aminés à chaîne ramifiée ainsi que par une baisse de la méthioninémie. Cette dernière suggère que ce composé est fourni en quantité insuffisante à l'organisme par le complément azoté alors que les prélèvements par la glande mammaire sont accrus (Rulquin, 1983). La méthionine peut donc être considérée comme limitante à ce stade de la lactation.

La mobilisation des protéines corporelles, appréciée par les teneurs sanguines en 3-méthylhistidine, paraît intense en première semaine de lactation; elle semble diminuer dès la $3^{e}$ semaine.

Cette étude, limitée aux 6 premières semaines de la lactation, a permis de montrer que la carence énergétique se manifestait, au moins jusqu'à la $3^{e}$ semaine, par une utilisation accrue des acides aminés à des fins énergétiques. La méthionine s'est révélée être le premier acide aminé limitant lorsque le besoin en PDI était satisfait.

Champredon C., Rémond B., Pion R., 1977. C.R. Soc. Biol., 171, 60-66.

Journet M., Faverdin P., Rémond B., Vérité R., 1983. Bull. Tech. C.R.Z.V. Theix I.N.R.A., 51, 7-17. Rulquin H., 1983. Reprod. Nutr. Dévelop., 23, 1029-1042. 